\title{
Causes of chronic cough in adolescent and adult patients
}

\author{
Xiaofang Gao*1 $\mathbb{D}$, Guangrong $\mathrm{Hu}^{2}$, Peng Yan $^{1}$ \\ Received: 6 Feb 2020 \\ Published: 9 Dec 2020
}

\section{Abstract}

Background: Adolescents differ from adults in certain aspects, but the differences in the etiology of chronic cough between adolescents and adults have not been specifically investigated. The purpose of this study is to evaluate the causes of chronic cough in adolescent patients in comparison with those in adult patients.

Methods: The electronic medical records were retrospectively screened for patients with the initial diagnosis of chronic cough from 2016 to 2018. Clinical variables and the causes of chronic cough were collected in patients who met inclusion criteria. Patients were assigned to adolescent (13 to 18 years) and adult ( $>18$ years) group based on age. The distribution of causes of chronic cough was compared by t-test and $\chi 2$ tests between adolescent and adult groups using SPSS. Significant level was set at 0.05.

Results: The enrolled patients consisted of 16 adolescents and 73 adults with chronic cough. The most common causes of chronic cough were cough-variant asthma and upper airway cough syndrome in both adolescents and adults. The frequency of post infectious cough in adolescents was significantly higher than that in adults $(12.5 \%$ vs. $1.36 \% ; \mathrm{p}=0.024)$. Although no significant difference between the two groups was found, there was a trend that suggested that habitual cough was more common in the adolescent group (6.25\%:1.36\%); while gastroesophageal reflux disease (GERD) did not show similar results (6.25\%:20.54\%).

Conclusion: There are moderate differences in the causes of chronic cough between adolescent and adult patients. Realizing those differences would be helpful for clinicians to establish an appropriate differential diagnosis and make referral decisions.

Keywords: Chronic cough, Adolescent, Post infectious cough

Conflicts of Interest: None declared

Funding: None

*This work has been published under CC BY-NC-SA 1.0 license.

Copyright $₫$ Iran University of Medical Sciences

Cite this article as: Gao X, Hu G, Yan P. Causes of chronic cough in adolescent and adult patients. Med J Islam Repub Iran. 2020 (9 Dec);34:166. https://doi.org/10.47176/mjiri.34.166

\section{Introduction}

Cough is the primary focus in ten percent of patients seen in the respiratory clinic (1). Chronic cough is defined as a cough lasting for more than 8 weeks, and it commonly affects physical, psychological, and social domains of health (2). Over the last decade, there have been a number of advances in the clinical approach to chronic cough, and the cause of chronic cough can be identified in $80-95 \%$ of

Corresponding author: Dr Xiaofang Gao, gaoxiaofang1977@163.com

1. Department of Respiratory Medicine, Chinese People's Liberation Army General Hospital, Beijing, China

2. Department of Respiratory Medicine, 989 Hospital of Chinese People's Liberation Army, Luoyang, China cases by using an anatomical diagnostic protocol (3).

Adolescents are not smaller adults, and they differ from the adults in certain aspects, for example, the immune system and reproductive organs (4). The importance of the differentiation of diagnostic evaluation and treatment of children with chronic cough from adults is increasingly recognized in clinical medicine, while the differences of

$\uparrow$ What is "already known" in this topic:

Recognizing the etiology of chronic cough would be helpful for empiric therapy and efficient referral. The most common causes of chronic cough in adults have been elucidated by previous studies, while the differences between adolescents and adults need to be clarified.

\section{$\rightarrow$ What this article adds:}

The present study suggested that moderate differences in the causes of chronic cough existed among adolescents and adults. The incidence of post infectious cough in adolescents was significantly higher than that in adults, and there was a trend that habitual cough was more common, while gastroesophageal reflux disease was less common in adolescents. 
chronic cough between adolescents and adults have not been specifically investigated. The aim of this study was to compare the frequency and spectrum of causes of chronic cough between adolescents and adults.

\section{Methods}

This study was conducted by a retrospective chart review of the patients presenting to the respiratory clinic or admitted to the respiratory department of 989 Hospital of Chinese People's Liberation Army from January 1, 2016, to June 30, 2018. Patients with the initial diagnosis of chronic cough in electronic medical records and the cause of chronic cough eventually being determined were included in the analysis. Exclusion criteria were age less than 13 years, cough duration less than 8 weeks, presenting with other symptoms and/or signs indicating underlying disorder, presence of pulmonary lesions on radiography, prior history of chronic respiratory disease to account for the cough, current smokers or ex-smokers of $<2$ years. The study protocol was approved by the Institutional Review Board of 989 Hospital of the Chinese People's Liberation Army.

The Clinical Practice Guidelines for Diagnosis and Management of Cough (2015) by the Chinese Thoracic Society (CTS) Asthma Consortium was applied to patients with a suspected diagnosis of chronic cough (5). The Chinese cough guidelines were based on the cough guidelines endorsed by the American College of Chest Physicians (ACCP), European Respiratory Society (ERS), Japanese Respiratory Society etc.; and it has essentially the same structure and content as these guidelines (6-8).

Cough intensity was evaluated using the cough symptom score described by Hsu et al., which is a rating scale that divides cough into six incremental scales, with zero being no cough and five being the worst score for a distressing cough that occurs most of the time (9). Cough variant asthma (CVA) was diagnosed when the patients presented an isolated non-productive cough without a history of wheezing or dyspnea, and the bronchodilation test was positive. Upper airway cough syndrome (UACS) was considered when the patients described the sensation of having something drip down into their throat; the physical examination found erythema and 'cobblestone' appearance of the posterior pharyngeal mucosa; sinus roentgeno- gram showing the presence of mucosal blur. The habitual cough was judged to be present when there were obvious psychosocial factors prior to cough, cough coexisted with depression or anxiety, and resolved with psychotherapy. The diagnosis of gastroesophageal reflux disease (GERD), eosinophilic bronchitis, postinfectious cough and angiotensin-converting enzyme (ACE) inhibitor-induced cough were made according to the above guidelines (5-8).

The patients who met the inclusion criteria were divided into two groups based on age: patients aged between 13 and 18 years were assigned to the adolescent group, and the others were assigned to the adult group. The distribution of causes and frequency of chronic cough were compared between adolescent and adult group. Continuous variables were expressed as means with standard deviations. Categorical values were expressed as proportions or percentages. To compare the two patients group, t-test and $\chi 2$ tests were used for normally distributed and nominal data between groups. Significance was defined as $\mathrm{P}$ value $<0.05$, and all tests were 2-sided. Data were analyzed using the SPSS statistical package (Version 20.0; Chicago, IL, USA).

\section{Results}

During the study period, 89 patients who met the inclusion criteria were included in the analysis. The enrolled patients consisted of 16 (17.98\%) adolescent patients, and $73(82.02 \%)$ adult patients. Sex distribution was comparable between adolescent and adult patients, and there were more females than males in both groups. The duration of cough persistence at their first visit of adult patients was nonsignificantly longer than adolescent patients. There was no significant difference in cough symptom score among adolescent and adult patients (Table 1).

The most prevalent causes of chronic cough in both adolescent and adult groups were CVA and upper airway cough syndrome. However, the incidence of postinfectious cough in adolescent patients was significantly higher than that in adult patients $(\mathrm{p}=0.024)$, and the constituent ratio was also different between those two groups. The associated factors of postinfectious cough in the adolescent and adult groups were virus (1 case vs. 1 case) and Bordetella pertussis ( 1 case vs. 0 case) infection. Although no significant difference between the two groups, there

Table 1. Characteristics of adolescents and adults with chronic cough

\begin{tabular}{lccc}
\hline Variable & Adolescent $\mathrm{n}=16$ & Adult $\mathrm{n}=73$ & $\mathrm{p}$ \\
\hline Male gender $(\%)$ & $7(43.75 \%)$ & $28(38.35 \%)$ & 0.714 \\
Age, yr & $15.5 \pm 1.6$ & $55.7 \pm 13.8$ & $<0.001$ \\
Duration of cough, mo & $3(2-18)$ & $5(2-64)$ & 0.295 \\
Cough symptom score & $3.1 \pm 1.3$ & $3.6 \pm 2.5$ & 0.696 \\
\hline
\end{tabular}

\begin{tabular}{|c|c|c|c|c|}
\hline Cause & Adolescent & Adult & $\chi^{2}$ & $\mathrm{p}$ \\
\hline CVA & $6(37.5)$ & $24(32.87)$ & 0.12 & 0.726 \\
\hline Upper airway cough syndrome & $4(25.0)$ & $18(24.65)$ & 0.00 & 0.973 \\
\hline GERD & $1(6.25)$ & $15(20.54)$ & 1.81 & 0.171 \\
\hline Eosinophilic bronchitis & $2(12.5)$ & $7(9.58)$ & 0.12 & 0.728 \\
\hline Postinfectious cough & $2(12.5)$ & $1(1.36)$ & 4.99 & 0.024 \\
\hline Habitual cough & $1(6.25)$ & $1(1.36)$ & 1.42 & 0.232 \\
\hline ACE inhibitor-induced cough & 0 & $6(8.21)$ & 0.40 & 0.528 \\
\hline
\end{tabular}

VCA, cough variant asthma; GERD, gastroesophageal reflux disease; ACE, angiotensin-converting enzyme. 
was a trend suggesting that habitual cough was more common in the adolescent group (6.25\%:1.36\%), while GERD was the opposite of it $(6.25 \%: 20.54 \%)$. There was no ACE inhibitor-induced cough in the adolescent group for the absence of hypertension in this population (Table 2).

\section{Discussion}

In many cases, the etiology of chronic cough is clearly declared in a patient's history or on physical examination findings. In other cases, however, the diagnosis remains clinically challenging. Information on the most common causes of chronic cough is valuable information for physicians both to direct empiric therapy and to guide referrals efficiently (10). Our study suggested that the most common likely causative factors of chronic cough in adults were CVA, upper airway cough syndrome and GERD, while GERD was replaced by eosinophilic bronchitis and postinfectious cough in the adolescent.

The results presented in this paper indicated that a moderate difference in the causes of chronic coughs, such as postinfectious cough and habitual cough, existed among adolescents and adults. The onset of cough with symptoms suggestive of an upper or lower respiratory tract infection raises the possibility of a postinfectious cough (11). Cough related to respiratory tract infections often resolves within 3 weeks. However, a cough takes several months to resolve in a small proportion of patients. Potential pathogens related to chronic cough include respiratory syncytial virus, parainfluenza virus, Mycoplasma pneumoniae, Chlamydia pneumoniae, and Bordetella pertussis (12).

Our results showed that clinicians should especially be alert to pertussis in adolescent patients with chronic cough. The efficacy of the pertussis vaccine declines over time so that children may become vulnerable 5-10 years after being vaccinated (13). Although immunization fails to protect them against pertussis; it does result in attenuated clinical features, which means that pertussis illness in adolescents is usually less severe, manifested by chronic cough accompanied by few other symptoms. Serology for pertussis toxin might be the best choice for the diagnosis of pertussis (14).

A previous study indicated that habitual cough was a common but baffling cause of cough in the school-aged child (15). Our study also showed there was a trend that habitual cough was more common in the adolescent group. Therefore, the clinicians ought to realize the difference of habitual cough among different age groups. Habitual cough is subject to psychological influences and more likely occurring under certain settings. This kind of cough does not respond to medications and occurs only during wakefulness and never during sleep (15). All of the associated etiologies must be exhaustively evaluated and treated before coming to the conclusion that the patient's cough is nonorganic. In cases in which habitual cough is suspected, a variety of interventions should be explored including psychotherapy, relaxation technique, breathing exercises, and speech therapy (16).

Because this is a retrospective study, there are limitations common to such a study design. For example, the present study is representative of a small group of adolescents with chronic cough, and further large-scale clinical trials with prospective design should be carried out to confirm our findings.

\section{Conclusion}

The frequency of the most common causes of chronic cough, CVA, and upper airway cough syndrome, are similar between adolescent and adult patients, but moderate differences such as postinfectious cough still exist among different age groups. Realizing those differences would be helpful for clinicians to establish an appropriate differential diagnosis and make referral decisions.

\section{Conflict of Interests}

The authors declare that they have no competing interests.

\section{References}

1. Long L, Lai K. Characteristics of Chinese chronic cough patients. Pulm Pharmacol Ther. 2019;57:101811

2. Iyer VN, Lim KG. Chronic cough: an update. Mayo Clin Proc. 2013;88(10):1115-1126.

3. Michaudet C, Malaty J. Chronic Cough: Evaluation and Management. Am Fam Physician. 2017;96(9):575-580.

4. Ioan I, Poussel M, Coutier L, Plevkova J, Poliacek I, Bolser DC, et al. What is chronic cough in children? Front Physiol. 2014;5:322.

5. Lai K, Shen H, Zhou X, Qiu Z, Cai S, Huang K, et al. Clinical Practice Guidelines for Diagnosis and Management of Cough-Chinese Thoracic Society (CTS) Asthma Consortium. J Thorac Dis 2018;10(11):6314-6351.

6. Irwin RS, Baumann MH, Bolser DC, Boulet LP, Braman SS, Brightling CE, et al. Diagnosis and management of cough executive summary: ACCP evidence-based clinical practice guidelines. Chest. 2006;129(1 Suppl):1S-23S.

7. Morice AH, Fontana GA, Sovijarvi AR, Pistolesi M, Chung KF, Widdicombe $\mathrm{J}$, et al. The diagnosis and management of chronic cough. Eur Respir J. 2004;24(3):481-492.

8. Committee for the Japanese Respiratory Society Guidelines for Management of Cough, Kohno S, Ishida T, Uchida Y, Kishimoto H, Sasaki $\mathrm{H}$, et al. The Japanese Respiratory Society guidelines for management of cough. Respirology. 2006;11 Suppl 4:S135-186.

9. Zhijing Wang, Miao Wang, Siwan Wen, Li Yu, Xianghuai Xu. Types and Applications of Cough-Related Questionnaires. J Thorac Dis. 2019;11(10):4379-4388.

10. Gibson P, Wang G, McGarvey L, Vertigan AE, Altman KW, Birring SS, et al. Treatment of Unexplained Chronic Cough: CHEST Guideline and Expert Panel Report. Chest. 2016;149(1):27-44.

11. Wang K, Fry NK, Campbell H, Amirthalingam G, Harrison TG, Mant $\mathrm{D}$, et al. Whooping cough in school age children presenting with persistent cough in UK primary care after introduction of the preschool pertussis booster vaccination: prospective cohort study. BMJ. 2014;348:g3668.

12. Perotin JM, Launois C, Dewolf M, Dumazet A, Dury S, Lebargy F, et al. Managing patients with chronic cough: challenges and solutions. Ther Clin Risk Manag. 2018;14:1041-1051.

13. Wang L, Lei D, Zhang S. Acellular pertussis vaccines in China Vaccine. 2012;30(50):7174-7178.

14. Ebell MH, Marchello C, Callahan M. Clinical Diagnosis of Bordetella Pertussis Infection: A Systematic Review. J Am Board Fam Med. 2017;30(3):308-319.

15. Haydour Q, Alahdab F, Farah M, Barrionuevo P, Vertigan AE, Newcombe PA, et al. Management and diagnosis of psychogenic cough, habit cough, and tic cough: a systematic review. Chest. 2014;146(2):355-372.

16. Wright MFA, Balfour-Lynn IM. Habit-tic cough: Presentation and outcome with simple reassurance. Pediatr Pulmonol. 2018;53(4):512516. 